\title{
Ultrafiltration d'émulsions huile-eau et de microémulsions de cyclohexane par un système à disque rotatif
}

\author{
Nadji Moulai-Mostefa ${ }^{1}$, Ambroise Brou ${ }^{2}$, Lu-Hui Ding ${ }^{2}$ et Michel Y. Jaffrin ${ }^{2, a}$ \\ 1 Centre Universitaire Yahia Fares, Ain D'Heb, 26001 Médéa, Algérie \\ 2 UMR CNRS 6600, Département Génie Biologique, UTC, BP 20529, 60205 Compiègne Cedex, France
}

Reçu le 28 mars 2003, accepté le 6 février 2004

\begin{abstract}
Résumé - La séparation de la phase huileuse à partir d'effluents huile-eau comportant des émulsions secondaires est très difficile par les procédés traditionnels et nécessite l'emploi de l'ultrafiltration. Des émulsions huile-eau contenant 2 et $4 \%$ d'huile ont été ultrafiltrées à l'aide d'un prototype de filtration dynamique comportant un disque tournant à grande vitesse près d'une membrane de $50 \mathrm{kDa}$ de $190 \mathrm{~cm}^{2}$ de surface. La turbidité de la solution initiale à $4 \%(21900 \mathrm{NTU})$ chute à 2 NTU dans le perméat après 15 min de filtration, ce qui représente une rétention de 99,5\% de l'huile. Les flux de perméat augmentent avec la vitesse de rotation du disque pour atteindre environ $901 . \mathrm{h}^{-1} \cdot \mathrm{m}^{-2}$ à $2000 \mathrm{tr} . \mathrm{min}^{-1}$ à $24{ }^{\circ} \mathrm{C}$. L'ajout d'ailettes de $6 \mathrm{~mm}$ de hauteur permet de doubler le flux de perméat à la même vitesse. Les microémulsions sont constituées de 4 à $20 \%$ de cyclohexane, 0,5 à $16 \%$ de 2-butanol et de 0,25 à $8 \%$ de dodecyl sulfate de sodium (SDS) dans de l'eau pure. Les flux de perméat, très élevés $\left(2001 \cdot \mathrm{h}^{-1} \cdot \mathrm{m}^{-2}\right)$ à $2000 \mathrm{tr} \cdot \mathrm{min}^{-1}$ avec un disque lisse pour la solution la plus diluée chutent à $15 \mathrm{l} \cdot \mathrm{h}^{-1} \cdot \mathrm{m}^{-2}$ pour la plus concentrée. L'ajout d'ailettes permet de doubler ces flux.
\end{abstract}

Mots clés : Ultrafiltration dynamique / émulsions / microémulsions / disque rotatif

\begin{abstract}
Ultrafiltration of oil-in-water emulsions and cyclohexane microemulsions using a rotating disk system. Oil separation from oil-water effluents containing secondary emulsions is very difficult by traditional techniques and requires using ultrafiltration through a membrane. Oil-water emulsions were ultrafiltered using a prototype of a rotating disk dynamic filtration system equipped with a $190 \mathrm{~cm}^{2}$ $50 \mathrm{kDa}$ circular membrane. The turbidity of initial solution at $4 \% \mathrm{wt}$ oil concentration (21900 NTU) dropped to $2 \mathrm{NTU}$ in the permeate after $15 \mathrm{~min}$ of filtration, representing an oil rejection coefficient of 99.5\%. Permeate fluxes increased with disk rotation speed to reach $90 \mathrm{l} \cdot \mathrm{h}^{-1} \cdot \mathrm{m}^{-2}$ at $2000 \mathrm{rpm}$ and $24{ }^{\circ} \mathrm{C}$. The addition of $6 \mathrm{~mm}$ vanes on the disk increased the permeate flux by $100 \%$ at the same speed. Micro emulsions were composed of 4 to $20 \%$ of cyclohexane, 0.5 to $16 \%$ of 2-butanol and 0.25 to $8 \%$ of dodecyl sodium sulphate (SDS) in pure water. Permeate fluxes, which were very high $\left(2001 . \mathrm{h}^{-1} \cdot \mathrm{m}^{-2}\right)$ at $2000 \mathrm{rpm}$ with a smooth disk and the most dilute solution, dropped to $151 . \mathrm{h}^{-1} \cdot \mathrm{m}^{-2}$ for the most concentrated one. Addition of vanes permitted to increase these fluxes by $100 \%$.
\end{abstract}

Key words: Dynamic ultrafiltration / emulsions / microemulsions / rotating disk

\section{Introduction}

Ces dernières années, de nombreux travaux ont été consacrés aux effluents industriels contenant des résidus huileux et à leur impact sur l'environnement. Ces rejets proviennent de plusieurs industries : mécanique, métallurgique et pétrolière. Ces industries génèrent de grandes quantités d'effluents huileux qui sont, dans la majorité des cas, rejetées dans la nature sans séparation de

\footnotetext{
a Auteur correspondant : michel.jaffrin@utc.fr
}

la phase huile par un traitement approprié. La présence de l'huile dans l'eau peut se manifester de trois manières : mélange immiscible, émulsions huile/eau instables et émulsions secondaires huile/eau très stables. Dans les deux premiers cas, la séparation de l'huile de l'eau est facile et nécessite l'utilisation de procédés mécaniques ou physico-chimiques. Cependant, dans les émulsions secondaires, la taille des gouttes d'huile est généralement inférieure à $20 \mu \mathrm{m}$, ce qui rend les processus classiques de séparation inefficaces. Les séparations membranaires, développées ces dernières années, constituent 


\section{Nomenclature}

\begin{tabular}{|ll|}
\hline$k:$ & coefficient d'entraînement du fluide par le disque \\
$N:$ & vitesse de rotation du disque $\left(\right.$ tr.min $\left.^{-1}\right)$ \\
$p:$ & pression $(\mathrm{Pa})$ \\
$p_{\mathrm{c}}:$ & pression périphérique $(\mathrm{Pa})$ \\
$p_{\mathrm{tm}}:$ & pression transmembranaire moyenne $(\mathrm{Pa})$ \\
$r:$ & distance radiale $(\mathrm{m})$ \\
$R:$ & rayon du disque $(\mathrm{m})$ \\
$\omega:$ & vitesse angulaire $\left(\mathrm{rad} \cdot \mathrm{s}^{-1}\right)$ \\
$\rho:$ & masse volumique $\left(\mathrm{kg} \cdot \mathrm{m}^{-3}\right)$ \\
$\nu:$ & viscosité cinématique $\left(\mathrm{m}^{2} \cdot \mathrm{s}^{-1}\right)$ \\
$\tau_{\mathrm{wt}}:$ & contrainte de cisaillement pariétale moyennée $(\mathrm{Pa})$ \\
\hline
\end{tabular}

une alternative de traitement pour la séparation des émulsions secondaires. L'ultrafiltration a ouvert notamment de bonnes perspectives pour le traitement des émulsions ou la récupération de produits organiques [1-5]. Le développement considérable dans la conception de nouvelles membranes dotées de propriétés particulières, a permis de généraliser ces techniques [6,7]. Néanmoins, les techniques de filtration classiques sont limitées par la polarisation de concentration à la surface de la membrane. Les solutés rejetés par la membrane s'accumulent à sa surface et forment une couche à forte concentration qui, augmente, d'une part la pression osmotique pour les petits solutés et, d'autre part la résistance à la filtration [8]. Le flux de perméat augmente avec la pression transmembranaire jusqu'à atteindre un plateau dont le niveau est fonction du taux de cisaillement.

Contrairement aux émulsions, les microémulsions sont thermodynamiquement très stables, ce qui rend leur cassage une opération très difficile. Ces systèmes nécessitent pour leur formulation de grandes quantités de tensioactifs et co-tensioactifs et sont capables d'émulsionner de grandes quantités d'huile. Peu de travaux [9] ont été consacrés à la séparation de ces systèmes qui présentent, à l'état actuel certaines limitations dues essentiellement à l'encrassement de la membrane par l'agent tensioactif qui est utilisé en grande quantité [10]. Récemment, de nouveaux types de membranes polymères sur support céramique ont été proposés $[11,12]$. Ces membranes combinent les avantages respectifs de chacun des matériaux, sélectivité et robustesse. Leur utilisation dans la séparation des microémulsions a conduit à une amélioration des taux de rejet de l'huile par rapport aux membranes dites classiques [13]. Ce résultat a été attribué au fait que les pores de la membrane diminuent lorsque les chaînes polymériques s'adsorbent à la surface solide de la membrane.

Pour diminuer les problèmes de colmatage rencontrés, il est nécessaire de créer à la surface de la membrane une contrainte de cisaillement importante sans pour autant augmenter le débit d'alimentation ou la pression transmembranaire. Plusieurs méthodes ont été proposées ces dernières années pour réduire la concentration de polarisation à la surface de la membrane : systèmes rotatifs $[14,15]$, injection de gaz [16]. Il semble que ces techniques dites dynamiques permettent de réduire le colmatage et d'augmenter le flux de perméat.

Cet article présente les résultats d'une étude expérimentale qui a porté sur l'ultrafiltration avec un système à disque rotatif d'une émulsion de fluide de coupe et d'une microémulsion à base de cyclohexane. Ce système fonctionne en régime de couche limite turbulente et le taux de cisaillement peut être augmenté pour une vitesse donnée en munissant le disque d'ailettes qui augmentent la vitesse du fluide entre le disque et la membrane $[17,18]$. L'objectif de notre étude est de déterminer l'influence de l'hydrodynamique (vitesse, pression, forme du disque) et des concentrations sur les performances du système en ultrafiltration d'émulsions et de microémulsions.

\section{Matériel et méthodes}

\subsection{Système de filtration}

Le banc de filtration (Fig. 1) comprend le module de filtration à disque rotatif alimenté par un réservoir de $5 \mathrm{l}$ au moyen d'une pompe. Celui-ci comporte une membrane circulaire de $15,5 \mathrm{~cm}$ de diamètre $\left(190 \mathrm{~cm}^{2}\right.$ de surface) dans un carter cylindrique en polyamide. Un disque métallique, lisse ou muni de 8 ailettes de $6 \mathrm{~mm}$ de hauteur, mis en rotation par un moteur électrique Leroy Somer tourne à une vitesse ajustable de 250 à 3000 tr.min $^{-1}$ à l'aide d'un variateur. Les pressions à l'entrée du module, à la périphérie du carter et à la sortie du rétentat sont mesurées par des capteurs de pression (Validyne DP 15). Le débit du perméat est mesuré par l'intermédiaire d'une balance électronique (Sartorius BP 3100) reliée, de même que les capteurs de pression, par une interface à un microordinateur. Les essais ont été conduits avec recyclage total du perméat et du rétentat pour garder une concentration constante.

Les membranes d'ultrafiltration (société ALTING, Strasbourg) sont en polyethersulfone (PES) avec un seuil de coupure de $50 \mathrm{kDa}$, comme celles utilisées par Dal-Cin et al. [14] et peuvent supporter jusqu'à $700 \mathrm{kPa}$ de pression et $75{ }^{\circ} \mathrm{C}$ au maximum. Le diamètre nominal des pores est de l'ordre de 8 à $10 \mathrm{~nm}$, donc inférieur à celui de la plupart des gouttelettes d'huile qui se situe entre 10 et $100 \mathrm{~nm}$. Leur perméabilité hydraulique est de $17 \mathrm{l} \cdot \mathrm{h}^{-1} \cdot \mathrm{m}^{-2} \cdot \mathrm{bar}^{-1}$. Une membrane neuve a été utilisée à chaque test.

\subsection{Produits utilisés}

Dans cette étude, un concentré de fluide de coupe a été utilisé pour préparer les émulsions. Il s'agit de l'huile de coupe Tasfalout fabriquée par la société NAFTAL (Algérie). Pour la préparation des microémulsions, la phase huileuse est constituée par du cyclohexane (société Sds, France), de pureté 99,5\% et de poids moléculaire 84,16 g.mole ${ }^{-1}$. Du dodecyl sulfate de sodium (SDS) est utilisé comme agent émulsifiant. Il possède une pureté de $95 \%$ et un poids moléculaire de 288,38 g.mole ${ }^{-1}$ 
Pompe péristaltique

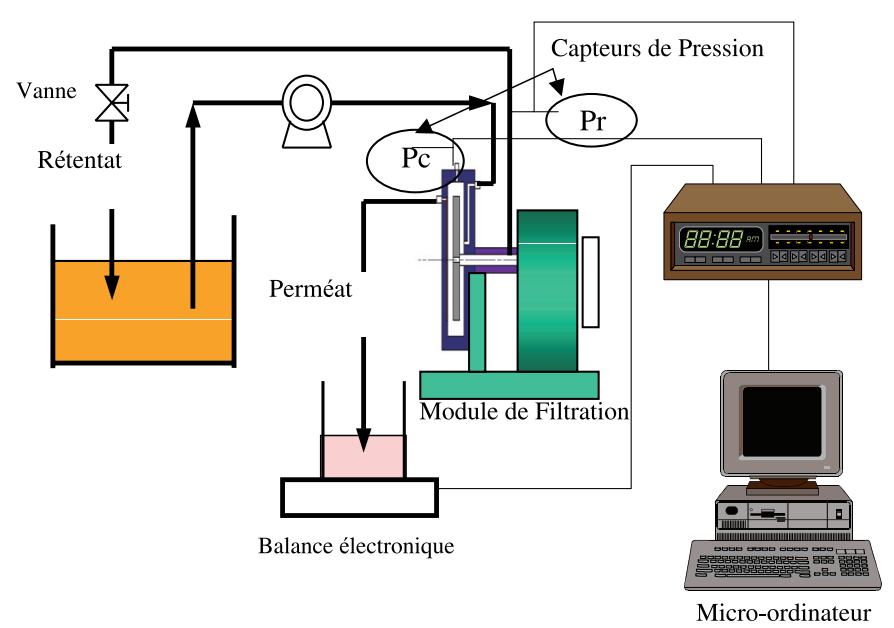

Fig. 1. Schéma de l'installation d'ultrafiltration.

(société Sigma). Le co-surfactant est du 2-butanol (société Aldrich) qui possède une pureté de $99 \%$ et un poids moléculaire de 74 g.mole ${ }^{-1}$. De l'eau déminéralisée a été utilisée pour la préparation des solutions.

\subsubsection{Préparation des émulsions d'huile de coupe}

Des émulsions commerciales d'huile de coupe ont été préparées en utilisant le concentré d'huile Tasfalout. Ce concentré contient une huile minérale de base naphténique $(80 \%)$, un tensioactif anionique (sulfonate de sodium, $10 \%$ ), un cotensioactif (alcool benzylique, $6 \%$ ) et d'autres additifs (4\%). Cette phase organique est diluée dans une phase aqueuse à des concentrations massiques de 2,5 et $4 \%$. Une concentration en huile de $4 \%$ correspond à l'ordre de grandeur des utilisations industrielles [19]. Les caractéristiques des émulsions préparées sont rassemblées dans le tableau 1. La turbidité de la solution est mesurée à l'aide d'un turbidimètre HACH, modèle 18900-10. Cette turbidité caractérise le trouble de la solution et est exprimée en NTU (Nephelometric Turbidity Unit). Si nécessaire la solution est diluée pour s'adapter à la gamme de l'appareil. Par des essais sur divers échantillons nous avons établi que cette turbidité, dans la gamme 1-25 NTU correspondant aux valeurs relevées sur le perméat, était proportionnelle à la concentration d'huile, 1 NTU étant équivalent à une concentration de $0,0065 \%$.

\subsubsection{Préparation des microémulsions}

Des microémulsions ont été préparées en mélangeant du cyclohexane (phase dispersée) et l'eau déminéralisée en présence d'un surfactant (SDS) et d'un co-surfactant (2-butanol). Le rapport co-surfactant/surfactant est maintenu constant et égal à 2, comme il est suggéré dans la littérature [9]. Les microémulsions préparées,
Tableau 1. Caractéristiques des émulsions préparées.

\begin{tabular}{cccccc}
\hline Émulsion & $\%$ masse & $\mathrm{pH}$ & $\begin{array}{c}\text { Viscosité } \\
(\mathrm{mPa} . \mathrm{s})\end{array}$ & $\begin{array}{c}\text { Turbidité } \\
(\mathrm{NTU})\end{array}$ & $\begin{array}{c}\text { Densité } \\
\left(\mathrm{kg} . \mathrm{m}^{-3}\right)\end{array}$ \\
\hline 1 & 2,5 & 8,64 & 13 & 10500 & 996 \\
2 & 4 & 8,50 & 13 & 21900 & 993 \\
\hline
\end{tabular}

qui présentent un comportement newtonien, ont été caractérisées du point de vue rhéologique à l'aide d'un rhéomètre de type Couette (Physica Rheolab MC1). La répartition des constituants de la microémulsion dans le perméat a été déterminée par un dosage chromatographique en phase gazeuse en utilisant un CPG PYE Unicam (modèle PU 4500, Philips). Les tensions de surface sont mesurées à l'aide d'un tensiomètre bidirectionnel Du Nouy (Model 70545, CSC Scientific Co, Inc.). La performance de perméation de la membrane a été évaluée en termes de flux de perméat et d'efficacité de séparation des constituants de la microémulsion. Les compositions des différentes solutions ainsi que leurs caractéristiques sont rassemblées dans le tableau 2.

\subsection{Hydrodynamique}

Le détail de l'écoulement entre le disque et la membrane a été étudié par Bouzerar et al. [17]. Le noyau non visqueux dans l'entrefer tourne à la vitesse angulaire $k \omega$ où $\omega$ désigne la vitesse angulaire du disque et $k$ le coefficient d'entraînement du fluide par le disque. La distribution de pression dans le noyau non visqueux obéit à la loi de Bernoulli

$$
p=1 / 2 \rho k^{2} \omega^{2} r^{2}+p_{0}
$$

où $p_{0}$ est la pression au centre ou celle dans la chambre quand le disque est immobile. Il est donc possible de déterminer ce coefficient $k$ par identification avec l'équation (1) en mesurant la pression périphérique $p_{\mathrm{c}}$ en $r=R$ (rayon intérieur de la chambre) à diverses vitesses de rotation. Pour un disque lisse, $k$ est égal à 0,45 , tandis qu'il atteint 0,84 pour un disque muni de 8 ailettes de $6 \mathrm{~mm}$ de hauteur [18]. Comme le perméat est recueilli à pression atmosphérique, la pression transmembranaire moyenne $p_{\text {tm }}$ s'obtient par intégration de $p$ sur la membrane pour donner :

$$
p_{\mathrm{tm}}=p_{\mathrm{c}}-1 / 4 \rho k^{2} \omega^{2} R^{2}
$$

La contrainte de cisaillement pariétale moyennée sur la surface de la membrane s'écrit, en régime turbulent [17]

$$
\tau_{\mathrm{wt}}=0,0164 \rho \nu^{0,2}(k \omega)^{1,8} R^{1,6}
$$

\section{Résultats}

\subsection{Ultrafiltration des émulsions d'huile de coupe}

\subsection{1 Évolution du flux au cours du temps et effet des ailettes}

La figure 2 présente la variation du flux de perméat au cours du temps pour deux types de disque et pour une 
Tableau 2. Compositions et propriétés physiques des microémulsions.

\begin{tabular}{ccccccc}
\hline Solution & $\begin{array}{c}\text { Cyclohexane } \\
(\%)\end{array}$ & $\begin{array}{c}\text { eau } \\
(\%)\end{array}$ & $\begin{array}{c}2 \text {-butanol } \\
(\%)\end{array}$ & $\begin{array}{c}\text { SDS } \\
(\%)\end{array}$ & $\begin{array}{c}\text { Viscosité } \\
(\mathrm{mPa} . \mathrm{s})\end{array}$ & $\begin{array}{c}\text { Densité } \\
\left(\mathrm{kg} . \mathrm{m}^{-3}\right)\end{array}$ \\
\hline I & 4 & 95,25 & 0,5 & 0,25 & 1,2 & 996 \\
II & 5 & 83 & 8 & 4 & 1,5 & 992 \\
III & 10 & 73,5 & 11 & 5,5 & 2,1 & 983 \\
IV & 20 & 56 & 16 & 8 & 3,1 & 982 \\
\hline
\end{tabular}

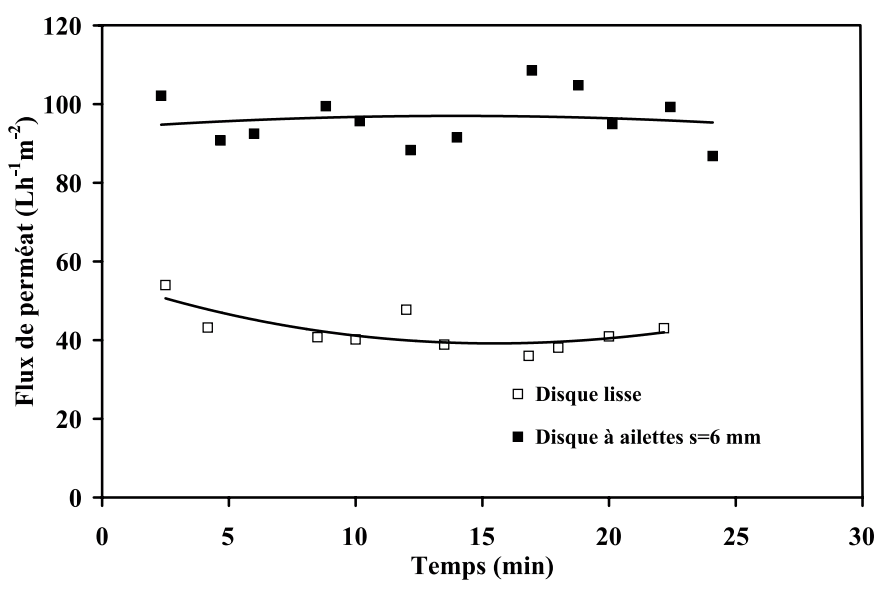

Fig. 2. Influence de la nature du disque sur l'évolution du flux en fonction du temps. Émulsion (4\%), Membrane PES $(50 \mathrm{kDa}), N=1000 \mathrm{tr} \cdot \mathrm{min}^{-1}, p_{\mathrm{tm}}=90 \mathrm{kPa}, T=23,7^{\circ} \mathrm{C}$.

concentration d'huile de $4 \%$ et une vitesse de rotation de 1000 tr. $\min ^{-1}$. La pression transmembranaire est maintenue constante à $90 \mathrm{kPa}$ par une vanne sur le rétentat et contrôlée par la mesure de la pression périphérique. Ainsi, on remarque que le flux diminue légèrement dans les premières minutes de l'opération, puis se stabilise à une valeur constante qui correspond à un équilibre de transfert de matière à la membrane. La stabilité du flux au cours du temps traduit la stabilité du colmatage de la membrane due au cisaillement élevé produit par la rotation du disque. On constate que l'utilisation d'un disque à ailettes de $6 \mathrm{~mm}$ de hauteur, permet d'augmenter le flux stabilisé de perméat d'environ $100 \%$. L'effet des ailettes se traduit par un meilleur entraînement du fluide et donc un cisaillement plus élevé suivant l'équation (3), qui limite la formation de dépôt à la surface de la membrane. La figure 3 montre l'évolution du flux et de la turbidité du perméat au cours du temps pour un autre essai avec le disque à ailettes et la même émulsion à $4 \%$. On remarque que le flux du perméat reste constant autour de $100 \mathrm{l} . \mathrm{h}^{-1} . \mathrm{m}^{-2}$ pour diminuer légèrement après $30 \mathrm{~min}$ indiquant un léger colmatage de la membrane par l'huile. La turbidité de la solution diminue dès les premières minutes de filtration après formation d'une couche de polarisation et reste très faible, inférieure à 2 NTU, contre 21900 NTU pour la solution initiale, tout au long de l'essai.

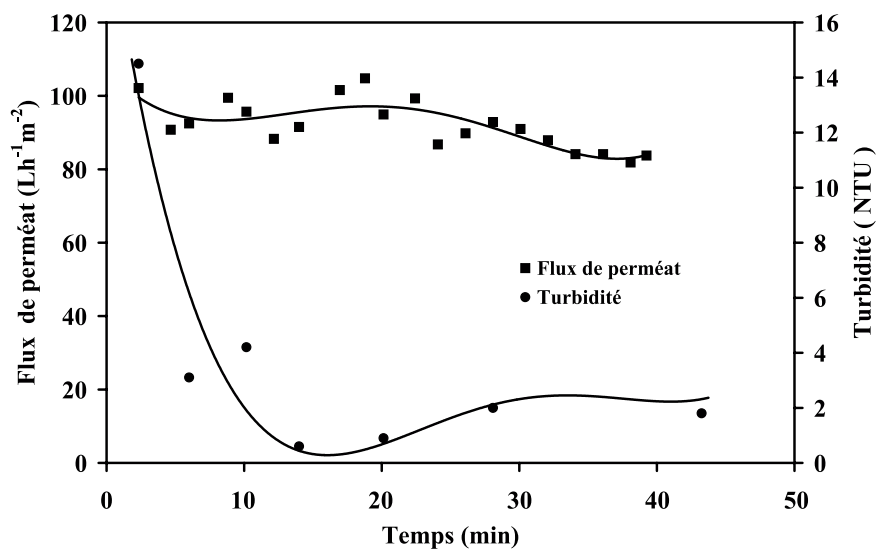

Fig. 3. Évolution du flux et de la turbidité en fonction du temps. Disque à ailettes, émulsion $(4 \%), N=1000$ tr.min ${ }^{-1}$, $T=23,7^{\circ} \mathrm{C}, p_{\mathrm{tm}}=90 \mathrm{kPa}$.

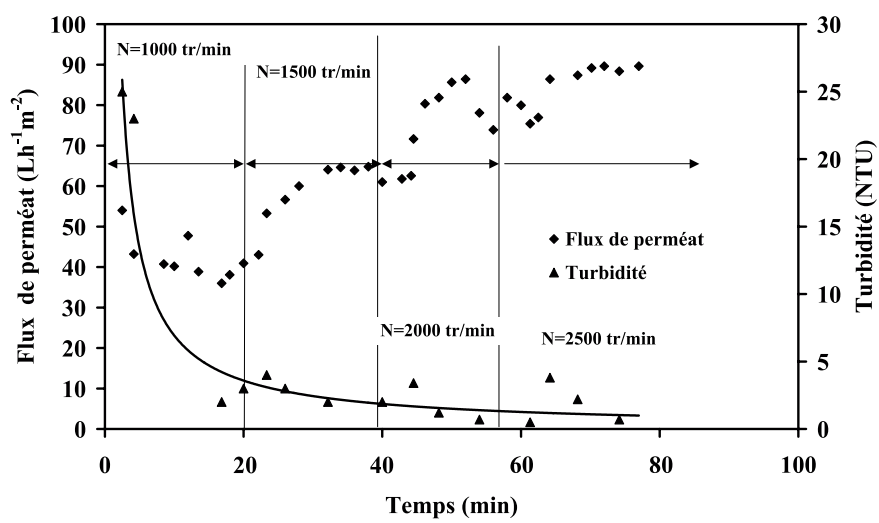

Fig. 4. Influence de la vitesse de rotation sur l'évolution du flux et de la turbidité en fonction du temps. Disque lisse, émulsion $(4 \%), N=1000$ tr.min ${ }^{-1}, T=23,7{ }^{\circ} \mathrm{C}, p_{\mathrm{tm}}=$ $90 \mathrm{kPa}$.

\subsubsection{Influence de la vitesse de rotation}

Ces essais ont été effectués avec la solution à $4 \%$ et avec un disque lisse en faisant varier la vitesse de 1000 à 2500 tr. $\min ^{-1}$, mais en gardant la même pression transmembranaire de $90 \mathrm{kPa}$ pour toutes les vitesses en ajustant la pression périphérique selon l'équation (2). Les variations du flux de perméat stabilisé, et de la turbidité en fonction du temps sont illustrées sur la figure 4. L'examen de cette figure montre que le flux de perméat augmente avec chaque augmentation de la vitesse de rotation du disque, mais progressivement. Cette progressivité, qui s'étale sur environ $10 \mathrm{~min}$, est due au temps nécessaire au cisaillement produit par cette augmentation de vitesse 


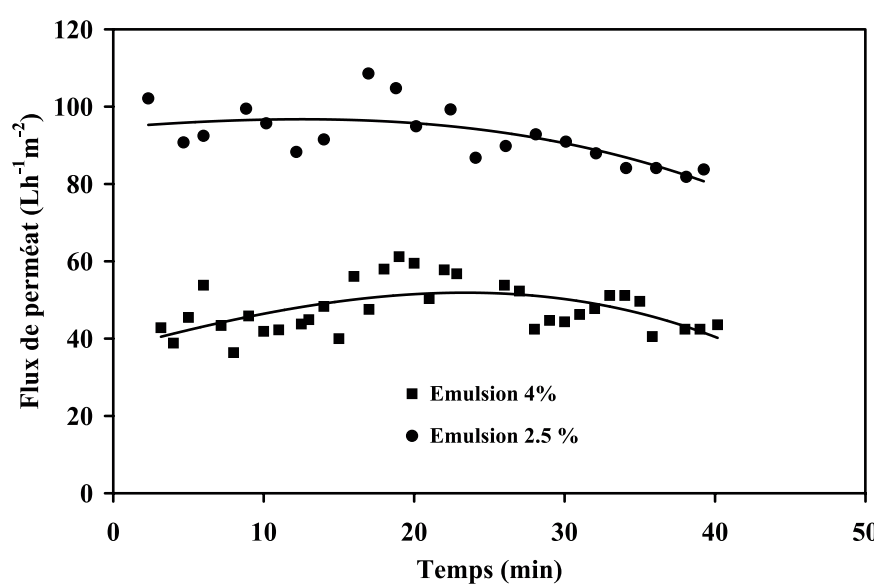

Fig. 5. Influence de la concentration de l'huile sur l'évolution du flux. Disque lisse, $N=1000$ tr. $\min ^{-1}, T=23,7^{\circ} \mathrm{C}, p_{\text {tm }}=$ $90 \mathrm{kPa}$.

pour réduire la couche de polarisation par un transfert de masse accru vers la solution. Par ailleurs, cet effet est moins visible à 2500 tr.min ${ }^{-1}$, car l'augmentation du flux est plus faible. La turbidité du perméat diminue rapidement jusqu'à 3 NTU en 20 min et reste très faible aux vitesses plus élevées. Des solutions très claires d'eau sont obtenues à la fin de l'opération d'ultrafiltration.

\subsubsection{Influence de la concentration initiale de l'émulsion}

Pour évaluer l'effet de concentration de l'émulsion, on a procédé à l'ultrafiltration de deux émulsions de concentrations en huile différentes, 2,5 et $4 \%$, avec un disque lisse. La figure 5 montre pour une même vitesse de rotation, l'évolution des flux de perméat au cours du temps. On note ainsi que le flux est deux fois plus important pour la solution à 2,5\% $\left(801 \cdot \mathrm{h}^{-1} \cdot \mathrm{m}^{-2}\right)$ que pour celle à $4 \%\left(401 \cdot h^{-1} \cdot \mathrm{m}^{-2}\right)$. Ceci peut être expliqué en partie par le modèle classique de polarisation de concentration de Blatt et al. [20], mais le facteur de réduction observé est plus élevé que ne le prédit ce modèle.

\subsubsection{Efficacité de séparation de I'huile}

À partir des mesures de turbidité du perméat, nous avons évalué les variations de la transmission de l'huile dans le perméat (pourcentage des concentrations résiduelles) en fonction du temps. Cette transmission, qui est très faible, chute rapidement pendant les premières 15 min de filtration (Fig. 6). Ensuite les valeurs résiduelles de l'huile dans le perméat restent très faibles, entre 0,01 et $0,02 \%$, ce qui correspond à un taux de rejet de l'huile supérieur à 99,5\%. Ce rejet est plus élevé pour un disque à ailettes. Les résultats obtenus avec notre système à disque rotatif sont généralement meilleurs que ceux obtenus par d'autres procédés pour une même pression transmembranaire, ainsi que le montre le tableau 3. L'injection d'un gaz conduit toutefois au même résultat que l'utilisation d'un

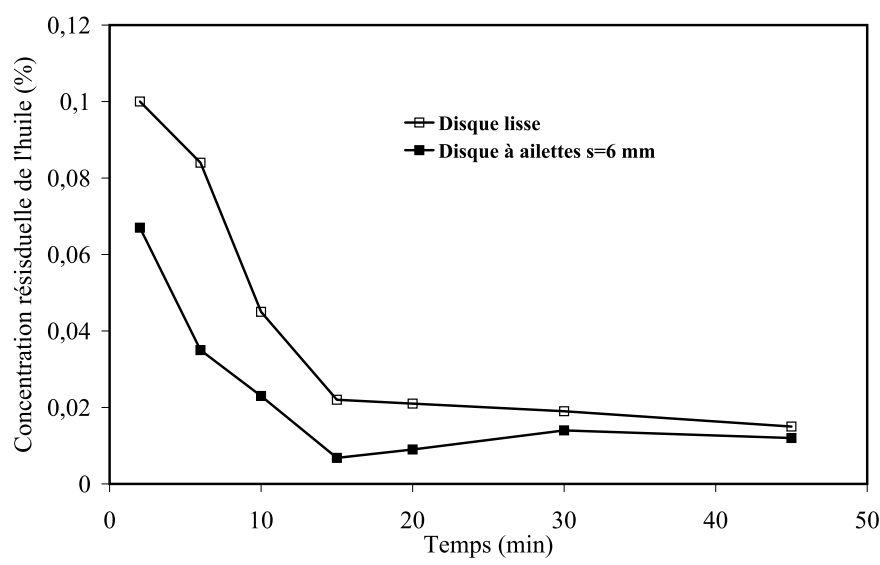

Fig. 6. Évolution de la concentration résiduelle de l'huile (\%) dans le perméat. Effet de la nature du disque, émulsion (4\%), $N=1000$ tr. $\min ^{-1}, p_{\text {tm }}=90 \mathrm{kPa}$.

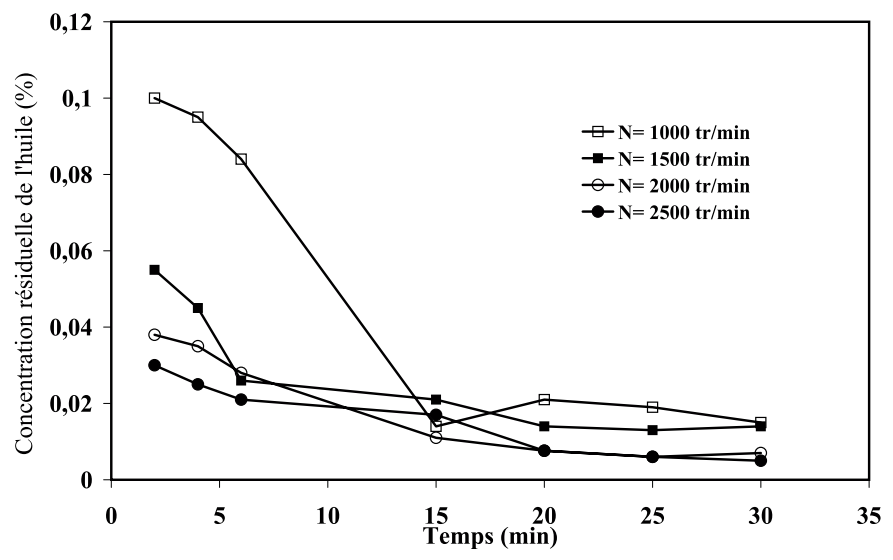

Fig. 7. Évolution de la concentration résiduelle de l'huile (\%) dans le perméat. Effet de la vitesse de rotation, disque lisse, émulsion $(4 \%), p_{\mathrm{tm}}=90 \mathrm{kPa}$.

disque rotatif à ailettes. La figure 7 montre également que la rétention de l'huile augmente avec la vitesse de rotation.

\subsection{Ultrafiltration de microémulsions}

\subsubsection{Effet de la vitesse de rotation et de la géométrie du disque sur le flux}

L'effet de la vitesse de rotation sur les flux d'ultrafiltration a été étudié pour le disque lisse avec une microémulsion très diluée (solution I). La pression à la périphérie du disque a été maintenue à $200 \mathrm{kPa}$ environ. La figure 8 montre que le flux augmente avec la vitesse de rotation du disque et est plus élevé que pour les émulsions pour atteindre des flux de $200 \mathrm{l} \cdot \mathrm{h}^{-1} \cdot \mathrm{m}^{-2}$. On trouve que le flux de perméat augmente avec la contrainte moyenne de cisaillement $\tau_{\mathrm{wt}}$ calculée par l'équation (3) suivant l'équation suivante :

$$
J=80,391 \tau_{\mathrm{wt}}^{0,25} \quad R^{2}=0,99
$$


Tableau 3. Comparaison des flux obtenus avec ce travail et des valeurs tirées de la littérature.

\begin{tabular}{lcccl}
\hline Référence & $\begin{array}{c}\text { Conc. Huil. } \\
(\% \text { masse })\end{array}$ & $\begin{array}{c}\text { Pression } \\
(\mathrm{kPa})\end{array}$ & $\begin{array}{c}\text { Flux } \\
\left(1 . \mathrm{h}^{-1} \cdot \mathrm{m}^{-2}\right)\end{array}$ & \multicolumn{1}{c}{ Configurations } \\
\hline Ce travail & 4 & 90 & $40-50$ & Disque lisse, $N=1500$ tr.min ${ }^{-1}$ \\
Ce travail & 4 & 90 & $90-110$ & Disque à ailettes, $N=1500$ tr.min $^{-1}$ \\
Dal-Cin [14] & 5 & 276 & 91 & Disque lisse, $N=1800$ tr.min \\
Lipp [21] & 5 & 100 & 40 & Cellule agitée \\
Mi-Jung Um [16] & 5 & 100 & $80-105$ & Injection de gaz \\
\hline
\end{tabular}

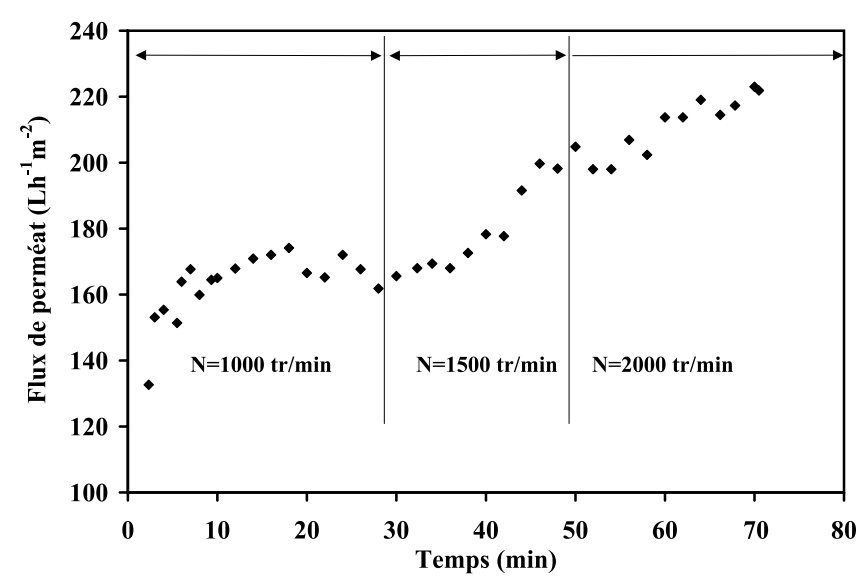

Fig. 8. Influence de la vitesse de rotation sur l'évolution du flux en fonction du temps. Disque lisse, microémulsion (I), $p_{\mathrm{tm}}=100 \mathrm{kPa}$.

Cette augmentation du flux est due au fait que la couche de polarisation créée par la coalescence des gouttelettes d'huile à la surface est déstabilisée par l'augmentation du taux de cisaillement imposé. Ceci démontre l'intérêt de la filtration dynamique par rapport aux procédés classiques qui permet d'atteindre des cisaillements très élevés avec des débits et des pressions relativement faibles.

La figure 9 montre l'effet de la géométrie du disque sur l'évolution du flux en fonction du temps pour la solution II seize fois plus concentrée en tensioactif que la solution I. Le disque à ailettes de $6 \mathrm{~mm}$ de hauteur donne un flux deux fois plus important que le disque lisse, mais ces flux sont beaucoup plus faibles que lorsqu'on utilise la solution I car ils sont limités par la concentration des constituants de la microémulsion.

\subsubsection{Influence de la concentration des constituants de la microémulsion}

L'augmentation de la concentration des constituants de la microémulsion a un effet très défavorable sur le flux de perméat (Fig. 10). Ces flux sont stables au cours du temps mais, malgré la présence d'ailettes, restent inférieurs à 50 l.h. ${ }^{-1} \cdot \mathrm{m}^{-2}$ pour la solution III et à $15 \mathrm{l} \cdot \mathrm{h}^{-1} \cdot \mathrm{m}^{-2}$ pour la solution IV. Par rapport à la solution I, le pourcentage en eau de la solution IV est passé de 95 à $56 \%$, celui en cyclohexane de 4 à $20 \%$, celui en

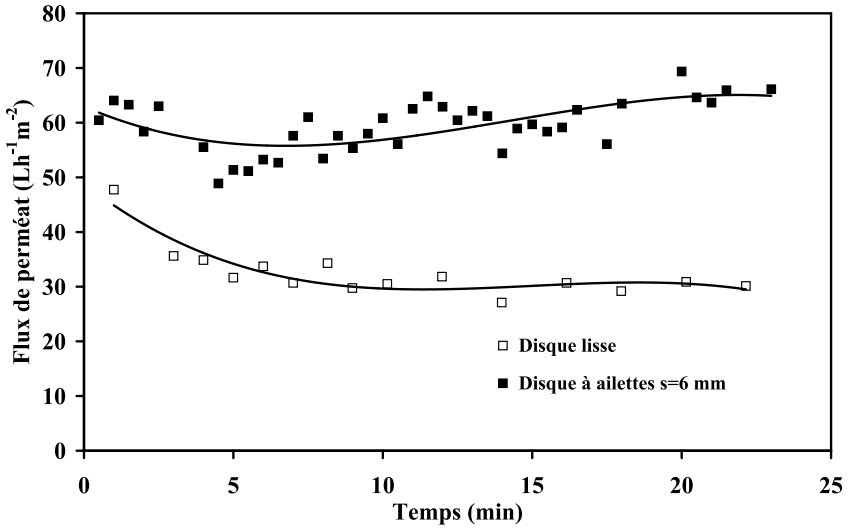

Fig. 9. Influence de la nature du disque sur l'évolution du flux en fonction du temps. Microémulsion (II), $N=1500$ tr.min $^{-1}$, $p_{\mathrm{tm}}=100 \mathrm{kPa}$.

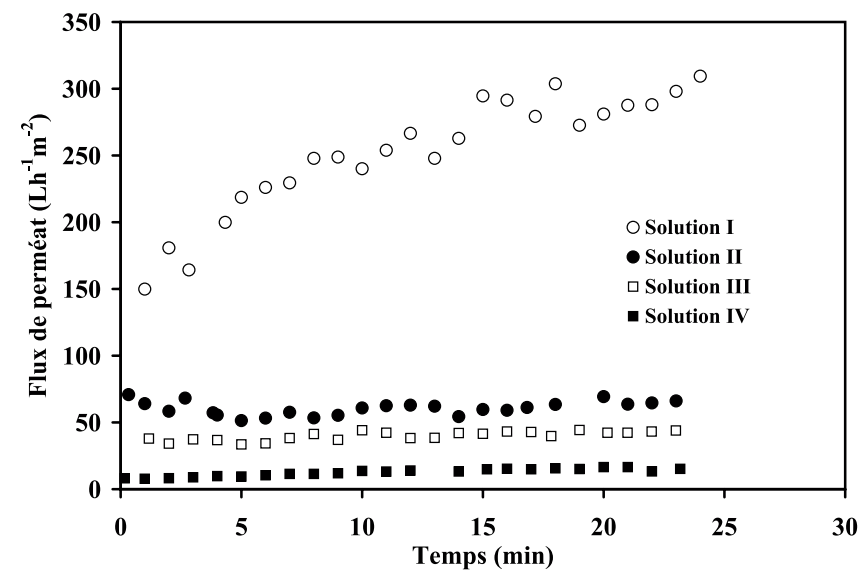

Fig. 10. Influence de la concentration de la microémulsion sur l'évolution du flux en fonction du temps. Disque à ailettes, $N=1500$ tr.min ${ }^{-1}, p_{\mathrm{tm}}=100 \mathrm{kPa}$.

2-butanol de 0,5 à $16 \%$ et celui du SDS de 0,25 à $8 \%$. La composition de la solution I a été choisie de telle sorte que les concentrations en surfactant et en co-surfactant soient très faibles.

\section{Discussion}

Selon les proportions des constituants, les microémulsions consistent en des dispersions de petites gouttelettes uniformes huile dans eau entourées d'une couche 
Tableau 4. Tensions de surface des solutions de perméat $\left(\mathrm{mN} \cdot \mathrm{m}^{-1}\right)$.

\begin{tabular}{|c|c|c|c|c|}
\hline $\begin{array}{l}\text { Conditions } \\
\text { de filtration }\end{array}$ & $\begin{array}{c}\text { Disque lisse } \\
\left(1000 \text { tr. } \text { min }^{-1}\right)\end{array}$ & $\begin{array}{c}\text { Disque lisse } \\
\left(1500 \text { tr.min }^{-1}\right)\end{array}$ & $\begin{array}{c}\text { Disque à ailettes } \\
(1000 \text { tr.min }\end{array}$ & $\begin{array}{c}\text { Disque à ailettes } \\
\left(1500 \text { tr.min }{ }^{-1}\right)\end{array}$ \\
\hline Solution I & 43,69 & 45,65 & 43,25 & 43,86 \\
\hline Solution II & 31,26 & 30,47 & 31,25 & 31,41 \\
\hline Solution III & 28,89 & 28,59 & 29,17 & 29,54 \\
\hline Solution IV & 25,82 & 25,55 & 25,35 & 25,35 \\
\hline
\end{tabular}

monomoléculaire de surfactants. La phase dispersée est composée de micelles de très faible diamètre. Selon Huibers et Shah [22], le diamètre des gouttelettes de microémulsions concentrées formulées avec du cyclohexane varie entre 10 et $100 \mathrm{~nm}$. Cette taille varie en fonction de la concentration en substances amphiphiles présentes dans la formulation. Il a été démontré par ailleurs que le diamètre des gouttelettes d'une miniémulsion est inversement proportionnel à la concentration en SDS [23]. Ceci suppose que la formule I est relativement instable par rapport aux autres formules puisque ces systèmes nécessitent généralement pour leurs formulations de grandes quantités de tensioactifs, alors que dans la solution I, la concentration en SDS est faible. Elle est légèrement supérieure à la concentration micellaire critique qui est de l'ordre de 0,23\% en masse. Dans ce cas, les micelles formées par les molécules du SDS sont sphériques [24]. La phase huileuse $(4 \%)$ est dissoute par les micelles et se retrouve dans la solution sous forme de gouttelettes relativement grandes. La filtration de la solution I a conduit à une déstabilisation rapide de la solution puisque les flux de permeat obtenus sont élevés. L'absence de colmatage de la membrane au bout de 20 min de filtration confirme ce mécanisme de déstabilisation qui est similaire à celui observé avec les émulsions. Cependant, lorsqu'on passe de la solution I à la solution II, le flux de perméat stabilisé a été divisé par un facteur 5,2. Dans cette solution, la concentration en tensioactif a été multipliée par un facteur 8 et celle de l'alcool par un facteur 16 . Les microémulsions obtenues avec cette deuxième solution sont très stables [9]. Dans ce type de formulation, la taille des gouttelettes est très petite et peut être du même ordre de grandeur que la taille des pores qui est d'environ $10 \mathrm{~nm}$ pour la membrane en PES choisie. Il est aussi fort probable que les tailles des gouttelettes dans la solution IV soient inférieures à celles de la solution II. Ces gouttelettes passent donc pour la plupart à travers les pores de la membrane. Leur passage forcé conduit systématiquement à un phénomène de coalescence qui dépend fortement de la taille des pores de la membrane [25]. Comme conséquence de ce phénomène, un film liquide d'huile se forme à l'intérieur des pores conduisant à leur colmatage. Ce colmatage est d'autant plus important que la solution est riche en tensioactifs et en cotensioactifs. Une chute de flux importante est obtenue comme conséquence de ce phénomène dans la solution IV où les concentrations des substances amphiphiles ont été multipliées par un facteur 32 (Fig. 10).

Il apparaît donc clairement que les facteurs limitatifs dans ce procédé sont les concentrations en tensioactif et en co-tensioactif. Cette réduction spectaculaire du flux
Tableau 5. Répartition de la composition des différents composants de la microémulsion rapportée à leur concentration globale dans le perméat.

\begin{tabular}{lc}
\hline Constituants & \% en masse \\
\hline SDS & 15 \\
2-butanol & 38 \\
Cyclohexane & 45 \\
\hline
\end{tabular}

peut être donc attribuée à la physicochimie de la solution au niveau de la membrane. Cette modification chimique de la surface est responsable du phénomène de colmatage induit d'une part par une polarisation de concentration par la présence de substances amphiphiles [26] et d'autre part, par la formation d'une couche coalescée à la surface $[10]$.

La mesure des tensions de surface des solutions de perméat de chaque type de microémulsion (Tab. 4) a montré que cette tension reste constante et ne dépend pas des conditions de filtration. Les mêmes valeurs sont obtenues pour une même microémulsion quelque soit le type de disque ou la vitesse de rotation utilisés. La répartition de la composition des constituants de la microémulsion concentrée rapportée à leur quantité globale dans le perméat (Tab. 5) confirme nos hypothèses de départ. Il semble qu'une bonne partie de la phase dispersée formée d'huile et d'alcool est passée à travers les pores de la membrane, ce qui expliquerait en outre pourquoi les tensions de surface sont faibles à la fin de l'opération de filtration. Cette perméation de la membrane est due aux tensions interfaciales très basses entre la couche dispersée à la surface de la membrane et la phase aqueuse continue formée d'eau et de tensioactifs [10]. Cependant, la concentration du SDS dans le perméat reste relativement faible en comparaison avec celle de l'alcool. Il devient donc clair que la limitation du flux peut être due en partie à la concentration élevée du SDS à la surface de la membrane. Les résultats obtenus en dynamique avec la membrane en PES sont comparables à ceux obtenus avec une membrane modifiée (CSP) pour l'ultrafiltration de microémulsions concentrées [10,13].

\section{Conclusion}

Cette étude a confirmé le potentiel du système de filtration dynamique à disque rotatif pour la déstabilisation des émulsions d'huile de coupe ou pour la séparation des composants d'une microémulsion. Les résultats expérimentaux sur le traitement de ce type de fluides ont 
permis de montrer que les performances de ce système pouvaient être améliorées par l'utilisation de disque muni d'ailettes et l'emploi de vitesses de rotation relativement élevées.

Les essais réalisés sur le traitement d'une émulsion d'huile de coupe ont montré que le flux stabilisé du perméat est augmenté de $100 \%$ lorsqu'on filtre avec un disque à ailettes par rapport à un disque lisse. L'effet des ailettes se traduit par un meilleur entraînement du fluide et donc un cisaillement important qui empêche la formation du dépôt à la surface de la membrane. L'augmentation de la vitesse de rotation de la membrane conduit à son tour à une augmentation du flux de perméat stabilisé. Ce flux est beaucoup plus important lorsqu'une solution diluée est traitée.

L'ultrafiltration des microémulsions a montré que l'efficacité du système de filtration est liée très fortement à la concentration des différents constituants. Pour les solutions diluées, les flux obtenus sont relativement élevés. L'augmentation de la vitesse de rotation ou l'utilisation du disque à ailettes conduit à une amélioration notable du flux de perméat. Cependant, lorsque les concentrations des composants de la microémulsion (huile, surfactant, cosurfactant) augmentent, on observe une forte diminution du flux. Le facteur limitatif dans ce procédé est la concentration du surfactant. Les flux obtenus par le procédé dynamique sont relativement élevés en comparaison par rapport à d'autres procédés, notamment avec l'utilisation du disque à ailettes. Par contre les rétentions par la membrane sont encore insuffisantes et il serait intéressant de faire des essais avec des membranes de seuil de coupure plus faible pour trouver le meilleur compromis entre rétention et flux de perméat.

Remerciements. Les auteurs remercient la société ALTING pour la fourniture des membranes.

\section{Références}

[1] S. Lee, Y. Aurell, H. Roques, Concentration polarisation membranes fouling and cleaning in ultrafiltration of soluble oil, J. Membr. Sci. 19 (1984) 23-28

[2] J. Mueller, Y. Cen, R.H. Davis, Cross flow microfiltration of oily water, J. Membr. Sci. 129 (1997) 221-235

[3] J.J. Kim, A. Chinen, H. Ohya, Membrane microfiltration of oily water, Macromol. Symp. 118 (1997) 413-418

[4] S.H. Lin, W.J. Lan, Waste oil/water emulsion treatment by membrane processes, J. Hazard. Mater. 59 (1998) 189-199

[5] I.W. Cumming, R.G. Holdich, I.D. Smith, The rejection of oil by microfiltration of a stabilised kerosene/water emulsion, J. Membr. Sci. 169 (2000) 147-155

[6] A. Hamza, V.A. Pham, T. Matsuura, J.P. Santerre, Development of membranes with low surface energy to reduce fouling in ultrafiltration applications, J. Membr. Sci. 131 (1997) 217-227

[7] P. Wang, N. XU, J. Shi, A pilot of the treatment of waste rolling emulsion using zirconia microfiltration membranes, J. Membr. Sci. 173 (2000) 159-166
[8] G. Belfort, J.M. Pimbley, A. Greiner, Y. Chang Kun, Diagnosis of membrane fouling using a rotating annular filter, J. Membr. Sci. 77 (1993) 1-22

[9] T. Aouak, S. Moulay, A. Hadj-Ziane, Microemulsion breakdown by pervaporation technique: the cyclohexane/water/n-butanol/sodium dodecylsulfate system, J. Membr. Sci. 173 (2000) 149-157

[10] R.S. Faibish, Y. Cohen, Fouling-resistant ceramicsupported membranes for ultrafiltration of oil-in-water microemulsions, J. Membr. Sci. 185 (2001) 129-143

[11] R.P. Castro, H.G. Monbouquette, Y. Cohen, Shearinduced permeability changes in polymer grafted silica membrane, J. Membr. Sci. 179 (2000) 207-220

[12] R.P. Castro, Development of ceramic-supported polymeric membranes for filtration of oil emulsions, Ph.D. Dissertation, University of California, Los Angeles, 1997

[13] R.S. Faibish, Y. Cohen, Fouling and rejection behavior of ceramic and polymer-modified ceramic membranes for ultrafiltration of oil-in-water emulsions and microemulsions, Colloids Surf. A 191 (2001) 27-40

[14] M.M. Dal-Cin, C.N. Licck, A. Kumar, S. Lealess, Dispersed phase back transport during ultrafiltration of cutting oil emulsions with a spinning membrane disc geometry, J. Membr. Sci. 141 (1998) 165-181

[15] R.C. Viadero, D.A. Masciola, B.E. Reed, R.L. Vaughan, Two-phase limiting flux in high-shear rotary ultrafiltration of oil-in-water emulsions, J. Membr. Sci. 175 (2000) $85-96$

[16] Mi-Jung UM, Seong-Hoon Yoon, Chung-Hak Lee, KunYong Chung, Jae-Jin Kim, Flux enhancement with gas injection in crossflow ultrafiltration of oily wastewater, Wat. Res. 35 (2001) 4095-4101

[17] R. Bouzerar, M.Y. Jaffrin, A. Lefèvre, P. Paullier, Concentration of ferric hydroxide suspensions in saline medium by dynamic cross-flow filtration, J. Membr. Sci. 165 (2000) 111-123

[18] A. Brou, L. Ding, P. Boulnois, M.Y. Jaffrin, Dynamic microfiltration of yeast suspensions using rotating disks equipped with vanes, J. Membr. Sci. 197 (2002) 269-282

[19] M. Belkacem, Nouvelle méthodologie dans le traitement des fluides de coupe par ultrafiltration, Thèse de Doctorat, INSA Toulouse, 1995

[20] W.F. Blatt, A. David, A.S. Michaels, L. Nelsen, Solute polarisation and cake formation in membrane ultrafiltration, In membrane science and technology, Flinn (ed.), Plenum, New York, 1970, pp. 47-49

[21] P. Lipp, C.H. Lee, A.G. Fane, C.J.D. Fell, A fundamental study of the ultrafiltration of oil-water emulsions, J. Membr. Sci. 36 (1988) 161-177

[22] P.D.T. Huibers, D.O. Shah, Evidence for synergism in non ionic surfactant mixtures. Enhancment of solublization in water-in-oil microemulsions, Langmuir 13 (1997) $5762-5765$

[23] N. Bechthold, F. Tiarks, M. Willert, K. Landfester, M. Antonietti, Miniemulsion polymerization: applications and new materials, Macromol. Symp., 151 (2000) 549-555

[24] R.J. Stokes, D.F. Evans, Fundamentals of interfacial engineering, Wiley, New York, 1997

[25] A. Hong, A.G. Fane, R. Burford, Factors affecting membranes coalescence of stable oil-in-water emulsions, J. Membr. Sci. 222 (2003) 19-39

[26] K. Mizoguchi, K. Fukui, H. Yanagishita, Ultrafiltration of a new type of non-ionic surfactant around the CMC, J. Membr. Sci. 208 (2002) 285-288 